Article

\title{
In Vitro Priming of Human T Cells by Dendritic Cells Provides a Screening Tool for Candidate Vaccines for Burkholderia pseudomallei
}

\author{
Durga Reddi ${ }^{1,+}{ }^{+}$Lydia Durant ${ }^{1} \odot$, David Bernardo ${ }^{1, \ddagger}$, Alistair Noble ${ }^{1,2}$, Nicholas R. English ${ }^{1}$, Philip Hendy ${ }^{1,3, \S}$, \\ Graeme C. Clark ${ }^{4}$, Joann L. Prior ${ }^{4}$, Ethel Diane Williamson ${ }^{4, *}$ and Stella C. Knight ${ }^{1}$
}

1 Antigen Presentation Research Group, Imperial Centre for Translational and Experimental Medicine, 72 Du Cane Road, London W12 0NN, UK; dreddi@parkinsons.org.uk (D.R.); 1.durant@imperial.ac.uk (L.D.); d.bernardo.ordiz@gmail.com (D.B.); Alistair.Noble@pirbright.ac.uk (A.N.); n.english@imperial.ac.uk (N.R.E.); p.hendy@imperial.ac.uk (P.H.); s.knight@imperial.ac.uk (S.C.K.)

2 Gut Microbes \& Health Program, Quadram Institute Bioscience, Norwich NR4 7UQ, UK

3 St Mark's Hospital, London North West University Healthcare NHS Trust, Harrow UT 84124, UK

4 Defence Science and Technology Laboratory, Porton Down SP4 0JQ, UK; gcclark@dstl.gov.uk (G.C.C.); jlprior@dstl.gov.uk (J.L.P.)

* Correspondence: dewilliamson@dstl.gov.uk

$\dagger$ Current Address: Parkinson's UK, London SW1V 1EJ, UK.

$\ddagger$ Current Address: Mucosal Immunology Lab, Instituto de Biología y Genética Molecular (IBGM), University of Valladolid, 47002 Valladolid, Spain.

check for updates

Citation: Reddi, D.; Durant, L.; Bernardo, D.; Noble, A.; English, N.R.; Hendy, P.; Clark, G.C.; Prior, J.L.; Williamson, E.D.; Knight, S.C. In Vitro Priming of Human T Cells by Dendritic Cells Provides a Screening Tool for Candidate Vaccines for Burkholderia pseudomallei. Vaccines 2021, 9, 929. https://doi.org/ $10.3390 /$ vaccines 9080929

Academic Editor: Steven B. Bradfute

Received: 24 June 2021

Accepted: 18 August 2021

Published: 22 August 2021

Publisher's Note: MDPI stays neutral with regard to jurisdictional claims in published maps and institutional affiliations.

Copyright: (c) 2021 by the authors. Licensee MDPI, Basel, Switzerland. This article is an open access article distributed under the terms and conditions of the Creative Commons Attribution (CC BY) license (https:// creativecommons.org/licenses/by/ $4.0 /)$.
$\S$ Current Address: Chelsea \& Westminster Hospital, Chelsea and Westminster Hospital NHS Foundation Trust, London SW10 9NH, UK.

Abstract: Murine dendritic cells, when pulsed with heat-killed Burkholderia pseudomallei and used to immunise naïve mice, have previously been shown to induce protective immunity in vivo. We have now demonstrated the in vitro priming of naïve human $\mathrm{T}$ cells against heat-killed B. pseudomallei, by co-culture with syngeneic B. pseudomallei-pulsed dendritic cells. Additionally, we have enriched the DC fraction such that a study of the differential response induced by pulsed DCs of either myeloid or plasmacytoid lineage in syngeneic human T cells was achievable. Whilst both mDCs and pDCs were activated by pulsing, the mDCs contributed the major response to B. pseudomallei with the expression of the migration marker CCR7 and a significantly greater secretion of the proinflammatory TNF $\alpha$ and IL1 $\beta$. When these DC factions were combined and used to prime syngeneic $\mathrm{T}$ cells, a significant proliferation was observed in the $\mathrm{CD}^{+}$fraction. Here, we have achieved human $\mathrm{T}$ cell priming in vitro with unadjuvanted B. pseudomallei, the causative organism of melioidosis, for which there is currently no approved vaccine. We propose that the approach we have taken could be used to screen for the human cellular response to candidate vaccines and formulations, in order to enhance the cell-mediated immunity required to protect against this intracellular pathogen and potentially more broadly against other, difficult-to-treat intracellular pathogens. To date, the polysaccharide capsule of B. pseudomallei, fused to a standard carrier protein, e.g., Crm, looks a likely vaccine candidate. Dendritic cells (DCs), providing, as they do, the first line of defence to infection, process and present microbial products to the immune system to direct downstream immune responses. Here, we have sought to use DCs ex vivo to identify immunogenic products from heat-killed B. pseudomallei. Using practical volumes of fresh human donor blood, we show that heat-killed B. pseudomallei activated and stimulated the expression of pro-inflammatory cytokines TNF- $\alpha$, IL- $1 \beta$ and IL- 6 from both myeloid and plasmacytoid DCs. Furthermore, B. pseudomallei-pulsed DCs cultured with naïve syngeneic $\mathrm{T}$ cells ex vivo, induced the activation and proliferation of the $\mathrm{CD} 4^{+} \mathrm{T}$-cell population, which was identified by cell surface marker staining using flow cytometry. Thus, both DC subsets are important for driving primary $\mathrm{T}$ helper cell responses to B. pseudomallei in healthy individuals and have the potential to be used to identify immunogenic components of B. pseudomallei for future therapies and vaccines. 


\section{Introduction}

Burkholderia pseudomallei (B.psm) is a Gram-negative infectious bacterium and the causative agent of melioidosis, a disease endemic to parts of south-east Asia. Disease may present as a chronic or acute infection of skin, lung, liver or spleen and can lead to pneumonia and septicaemia [1]. After initial exposure, B.psm replicates intracellularly within epithelial or phagocytic cells and can evade host immunity [2]. Due to the multiple routes of exposure, active efflux of antibiotics by the bacteria and the lack of an approved vaccine, infection with B.psm is problematic to manage. To date, the polysaccharide capsule of $B$. pseudomallei, fused to a standard carrier protein, e.g., $\mathrm{Crm}$, has been pursued as a promising vaccine candidate. In order to eradicate this intracellular infection, it will be essential for melioidosis vaccine candidates to induce effective cellular immunity. Dendritic cells (DCs) have a key role in the induction of cellular immune responses, by processing and presenting microbial products to the immune system to direct downstream immune responses. DCs express pattern recognition receptors (PRR) that recognise the pathogen-associated molecular patterns (PAMPs) within microbes. DCs internalise microbial components by receptor-mediated endocytosis, then process and present antigens associated with major histocompatibility complex (MHC) class II to $\mathrm{CD}^{+}$or MHC class I to $\mathrm{CD} 8^{+} \mathrm{T}$ cells residing in local lymph-nodes. Upon stimulation, DCs also express CCR7, allowing their migration to local lymph-nodes, and maturation markers including CD80, CD86 and CD40, which provide co-stimulatory signals to T cells. Finally, DCs secrete a variety of cytokines that direct specific T-cell responses.

The two major subsets of DCs in humans are myeloid and plasmacytoid. Myeloid DCs (mDCs) are typically responsive to bacteria, whereas plasmacytoid DCs (pDCs) respond to viruses [3], although pDCs can be activated by some bacteria including Staphylococcus aureus and Streptococcus pyogenes $[4,5]$. Moreover, $\mathrm{pDC}$ co-cultured with $\mathrm{mDC}$ responsive to bacterial stimulation show a unique capacity to mature and be activated, suggesting synergy between the two subsets [6]. Human and murine pDCs internalise and cause the killing of B.psm [7]. Furthermore, the passive transfer into mice of bone-marrow (BM)derived DCs that had been pulsed with heat-killed B. pm and matured in the presence of $\mathrm{CpG}$, induced protective immunity against subsequent exposure to B.psm, demonstrating the significant role for DCs in driving protective cell-mediated immune responses [8].

The focus of this study was to investigate whether a primary human T-cell response to B.psm could be induced in vitro by co-culture with syngeneic DC, in order to establish a system to analyse a protective human cell-mediated response to the bacterium. To achieve this and using practical volumes of fresh human donor blood, we have enriched for DCs and pulsed these with heat-killed B.psm K96243, the genome-sequenced strain of B.psm [1]. A sufficient quantity of DCs were derived to enable the separation of myeloid and plasmacytoid subsets and study of their phenotype (activation and cytokine expression) and function (ability to stimulate primary T-cell responses) after pulsing with B.psm. We have found that the major response to unadjuvanted and heat-killed B.psm came from the myeloid population. When the total DC faction was pulsed with B.psm prior to co-culture with syngeneic naive $\mathrm{T}$ cells, a significant proliferation of the gated CD4+ population was observed, together with the detection of $\mathrm{T}$ cell activation markers, indicating in vitro priming. We propose that this in vitro human cell system may be a useful approach for screening candidate vaccines in order to enhance a protective CMI response. Furthermore, our methodology could be used to assess memory responses in B.psm-exposed individuals. 


\section{Methods}

\subsection{Bacterial Preparation}

B. pseudomallei $\mathrm{K} 96243$ was cultured in L-broth and on L-agar at $37^{\circ} \mathrm{C}$. Bacterial stocks were maintained at $-80{ }^{\circ} \mathrm{C}$ as $20 \%$ glycerol suspensions. B. pseudomallei $\mathrm{K} 96243$ was handled at Advisory Committee for Dangerous Pathogens (ACDP) containment level 3. The bacteria were heat-killed as previously reported [8].

\subsection{Healthy Donors}

Blood (30-50 mL) was collected from healthy volunteers with no known autoimmune or inflammatory diseases, allergies or malignancies. Ethical approval was obtained from the Health Research Authority UK and London Brent Research Ethics Committee (05.Q0405.71). Written informed consent was received from participants prior to inclusion. For each independent experiment, a minimum of 3 and a maximum of 6 donors were used, as specified in the Figure legends.

\subsection{Dendritic Cell Enrichment}

Peripheral blood mononuclear cells (PBMCs) were isolated by centrifugation over Ficoll-Paque Plus (Amersham Biosciences, Chalfont St. Giles, UK). Total mDCs and pDCs were negatively enriched from PBMCs using the EasySep ${ }^{\mathrm{TM}}$ Human Pan-DC PreEnrichment Kit (StemCell Technologies, Cambridge, UK) according to manufacturer's instructions. Compared to whole PBMCs, where DCs (HLA-DR ${ }^{+}$and lineage (CD3/CD14/ CD16/CD19/CD34) ${ }^{-}$cells) are between $1-2 \%$ of live cells, the purity of DCs (mDCs and pDCs) after enrichment was between $65-70 \%$ of live cells. Enriched DCs were cultured in Dutch modified RPMI 1640 (Sigma-Aldrich, Dorset, UK) containing 100 U/mL penicillin/streptomycin, $2 \mathrm{mM}$ L-glutamine, $50 \mu \mathrm{g} / \mathrm{mL}$ gentamicin (Sigma-Aldrich) and 10\% foetal calf serum (TCS cell works, Buckingham, UK).

\subsection{Bacterial Stimulation}

Enriched DCs $\left(5 \times 10^{4}\right)$ were stimulated with ascending concentrations of B.psm or medium only (untreated control) for $0,3,20$ or $24 \mathrm{~h}$ at $37{ }^{\circ} \mathrm{C}$ and $5 \% \mathrm{CO}_{2}$. For DCs intracellular cytokine analysis, stimulation with $100 \mathrm{ng} / \mathrm{mL}$ of E. coli LPS (Sigma-Aldrich, Dorset, UK) was a positive control.

\subsection{Cell-Surface Antibody Labelling}

After 20-hour incubation, DCs were washed with FACS buffer (1x PBS containing 5\% FCS, $1 \mathrm{mM}$ EDTA and $0.02 \%$ sodium azide) and labelled with Near-IR Live/Dead Fixable Dead Cell stain (ThermoFischer Scientific) according to kit instructions. Cells were then labelled for $20 \mathrm{~min}$ on ice with antibodies, fixed with $1 \%$ paraformaldehyde in $0.85 \%$ saline and stored at $4{ }^{\circ} \mathrm{C}$ until flow cytometric analysis. "Fluorescence minus one" controls were used to determine positive staining for each marker.

\subsection{Intracellular Cytokine Analysis}

After 3-hour culture, DCs were harvested, labelled with viability stain and surface antibodies as above and fixed and permeabilised using Leucoperm A and B reagents (Bio-Rad, Watford, UK). Cells were labelled intracellularly with antibodies in Leucoperm B. Positive staining for all cytokines was determined by comparing to an untreated control.

\subsection{Enzyme Linked Immunosorbent Assay (ELISA)}

Enriched DCs were cultured with B.psm for $24 \mathrm{~h}$ and supernatants stored at $-20{ }^{\circ} \mathrm{C}$. Concentrations of IL-6, IL-1 $\beta$ and TNF- $\alpha$ were measured using DuoSet ELISA kits (R\&D Systems, Abingdon, UK) as per manufacturers' instructions. 


\subsection{DC: T Cell Co-Cultures}

Enriched DCs were stimulated with B.psm for $20 \mathrm{~h}$. Total $\mathrm{T}$ cells were purified from the same donor's PBMCs using the EasySep ${ }^{\mathrm{TM}}$ Human T cell Isolation kit (StemCell Technologies). Purity of total $\mathrm{CD}^{+}$and $\mathrm{CD} 8^{+} \mathrm{T}$ cells was estimated at $94 \%$. After 20-hour culture, T cells were labelled with Cell Trace Violet (CTV) dye (Thermo Fisher) as per manufacturer's instructions. CTV-labelled T cells $\left(2.5 \times 10^{5}\right)$ were co-cultured with either $10 \%\left(2.5 \times 10^{4}\right)$ or $5 \%\left(1.25 \times 10^{4}\right)$ B.psm-pulsed DCs for 7 days. As a positive control, $2.5 \times 10^{5}$ CTV-labelled T cells were stimulated with $5 \mu \mathrm{g} / \mathrm{mL}$ of plate-bound anti-CD3 (300331, Biolegend) and $5 \mu \mathrm{g} / \mathrm{mL}$ of soluble anti-CD28 (302913, Biolegend, London UK). CTV-labelled T cells cultured in medium only were an unstimulated control.

\subsection{Flow Cytometry Analysis}

Cells were acquired on the FACSCanto II Flow Cytometer (BD Biosciences) and analysed using FlowJo software version 10.

\subsection{Statistical Analysis}

One-way or two-way ANOVA followed by Dunnett's multiple comparisons test was used to compare experimental groups to medium-only controls. A Student's paired $t$-test was used to compare T-cell proliferation in the B.psm group to untreated control. Statistical analysis used GraphPad Prism version 7.0.

\section{Results}

\subsection{B. pseudomallei Activates Both $m D C s$ and $p D C s$ Enriched from Fresh Blood}

Immunisation with heat-killed B.psm protects mice against a subsequent pathogen challenge, suggesting that the killing of the bacteria preserves protective motifs and antigens [9]. To define an optimal non-toxic concentration of B.psm, DCs derived from fresh naive PBMCs were incubated with ascending concentrations of B.psm for $20 \mathrm{~h}$, with a baseline $0 \mathrm{~h}$ time-point used for comparison. To enable this and subsequent analyses, DCs were enriched from PBMCs such that, whereas DCs (HLA-DR ${ }^{+}$and lineage (CD3/CD14/CD16/CD19/CD34) cells) usually comprise between $1-2 \%$ of live cells, the content of DCs (mDCs and pDCs) after enrichment was between $65-70 \%$ of live cells (Figure 1A,B).

A
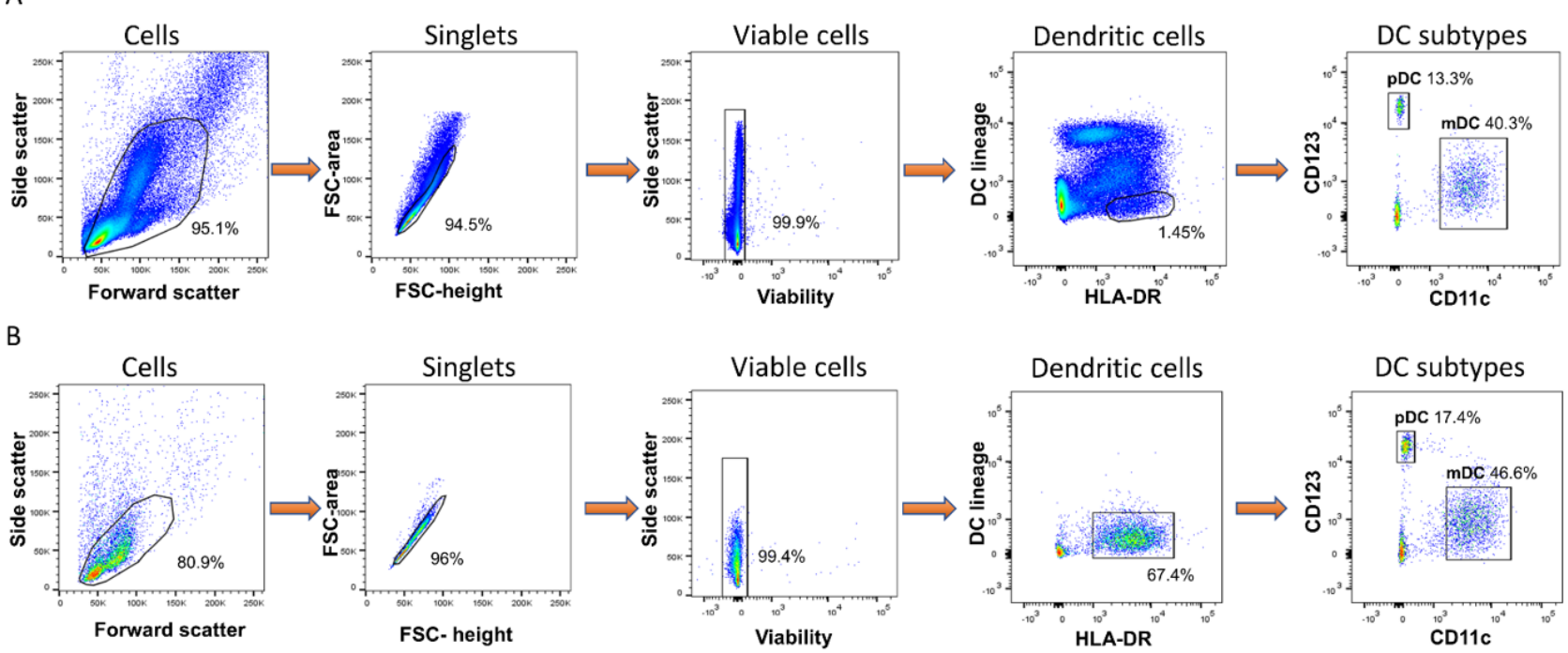

Figure 1. Enrichment of human blood myeloid and plasmacytoid dendritic cells from fresh PBMC. DCs were identified using side/forward scatter properties, doublet discrimination and gating on viable cells. Cells that were HLA-DR ${ }^{+}$and lineage ${ }^{-}\left(\mathrm{CD} 3, \mathrm{CD} 14, \mathrm{CD} 16, \mathrm{CD} 19\right.$ and CD34) were further divided into $\mathrm{mDCs}\left(\mathrm{CD} 11 \mathrm{c}^{+} \mathrm{CD} 123^{-}\right)$and $\mathrm{pDCs}\left(\mathrm{CD} 11 \mathrm{c}^{-} \mathrm{CD} 123^{+}\right)$. (A) DC identification within PBMC population before DC enrichment. (B) DC identification after enrichment. 
Using these enriched populations, the expression of activation markers CD80, CD86 and CD40 and lymph node migration marker CCR7 were measured on mDCs and pDCs using flow cytometry. The proportion of mDCs expressing CD80, CD86 and CD40 was significantly increased in response to high concentrations of B.psm at $20 \mathrm{~h}$ (Figure 2A). The highest concentration of B.psm also stimulated a significant increase in the proportion of pDCs expressing CD80, but not CD86 or CD40 (Figure 2B). Whilst the pDCs were less responsive than the mDCs, the median fluorescence intensity (MFI) of HLA-DR (MHCII) on both the mDCs and the pDCs increased significantly after $20 \mathrm{~h}$ of B.psm incubation (Figure 2C). Further, the proportion of $\mathrm{mDC}$ expressing CCR7 increased in all groups at $20 \mathrm{~h}$, whilst the proportion of pDCs expressing CCR7 was lower at $20 \mathrm{~h}$ in all groups; however, these changes were not statistically significant (Figure 2C). Thus, we determined that the highest concentration of $1 \times 10^{6} \mathrm{CFU} / \mathrm{mL}$ of B.psm optimally activated both DC subsets and was used in subsequent experiments.

A
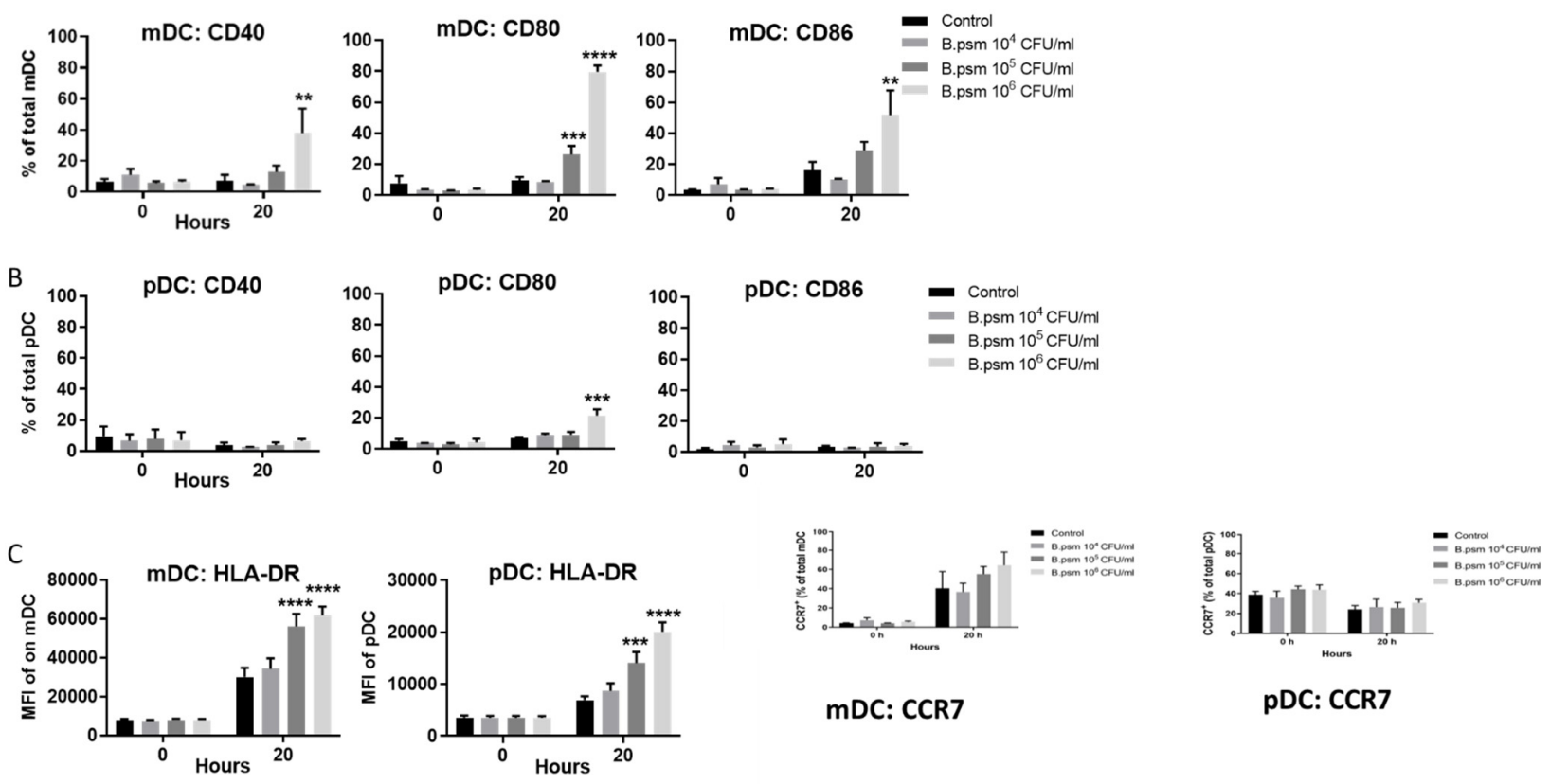

Figure 2. B. pseudomallei stimulates maturation and cytokine production by myeloid and plasmacytoid DC in healthy donors. Enriched DCs from healthy donor PBMCs were stimulated for $20 \mathrm{~h}$ with B. pseudomallei (B.psm) and percentage of cells expressing CD80, CD86 and CD40 was measured for (A) myeloid (m)DCs and (B) plasmacytoid (p)DCs using flow cytometry. (C) The median fluorescence intensity (MFI) of HLA-DR on mDCs and pDCs was also determined at $20 \mathrm{~h}$. The expression of lymph-node homing marker CCR7 on mDCs and pDCs subsets was also determined at $20 \mathrm{~h}$. Bars represent mean values \pm SEM from three (A,B) or six (C) independent experiments. A two-way (ANOVA) followed by Dunnett's multiple comparisons test was used to compare experimental groups to the medium only control. ${ }^{* *} p<0.01,{ }^{* * *} p<0.001$, $* * * * p<0.0001$.

\subsection{B.psm Stimulates Proinflammatory DC Cytokine Production}

Activated DCs secrete a variety of polarising cytokines that direct protective immune responses. We investigated the production of TNF- $\alpha$, IL- $1 \beta$ and IL- 6 by DCs in response to B.psm. At baseline, there was no detectable TNF- $\alpha$, IL-1 $\beta$ or IL- 6 in supernatants of stimulated DCs (Figure 3A). However, after $24 \mathrm{~h}$ of pulsing with B.psm, TNF- $\alpha$ and IL-6 were significantly increased (Figure 3A). To determine which DC subset was synthesising these cytokines, we stimulated DCs for $3 \mathrm{~h}$ with B.psm and measured the intracellular cytokines in the $\mathrm{mDC}$ and the $\mathrm{pDC}$ subsets. B.psm stimulation led to a significant increase in the proportion of $\mathrm{mDC}$ s expressing TNF- $\alpha$ and IL- $1 \beta$, while the proportion of $\mathrm{mDCs}$ expressing IL-6 was only significantly increased by E. coli LPS (Figure 3B). B.psm also stimulated a significant increase in the proportion of pDCs expressing TNF- $\alpha$ (Figure 3C). 
The proportion of mDCs or pDCs expressing TNF- $\alpha$ was similar (approximately $20 \%$ ), but the MFI of TNF- $\alpha$ was significantly higher in the mDCs compared to the $\mathrm{pDCs}$ in response to both B.psm (1.78-fold higher) and E. coli LPS (1.46-fold higher) stimulation (Figure 3C).
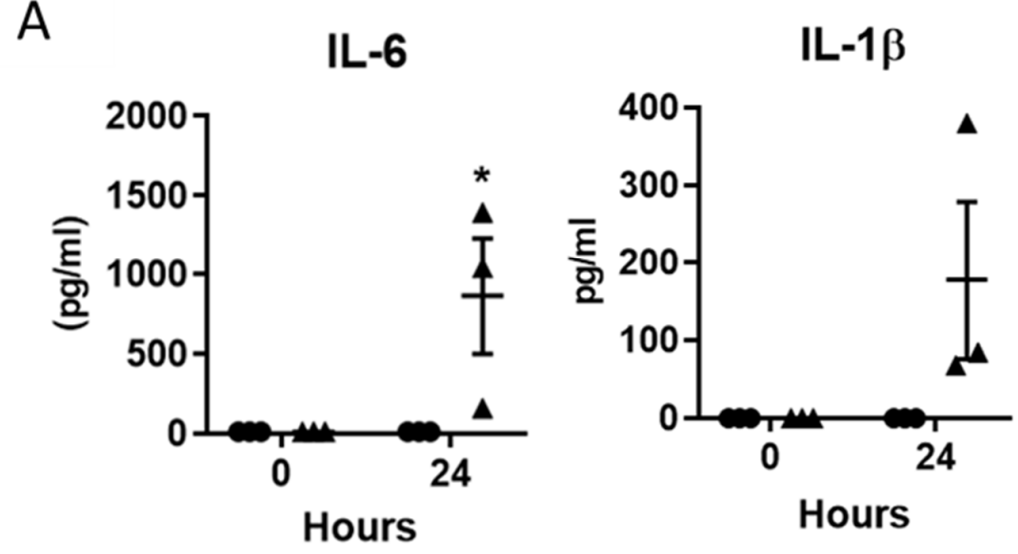

Control \ B.psm $10^{6} \mathrm{CFU} / \mathrm{ml}$

B

mDC: IL-6

mDC: IL-1 $\beta$
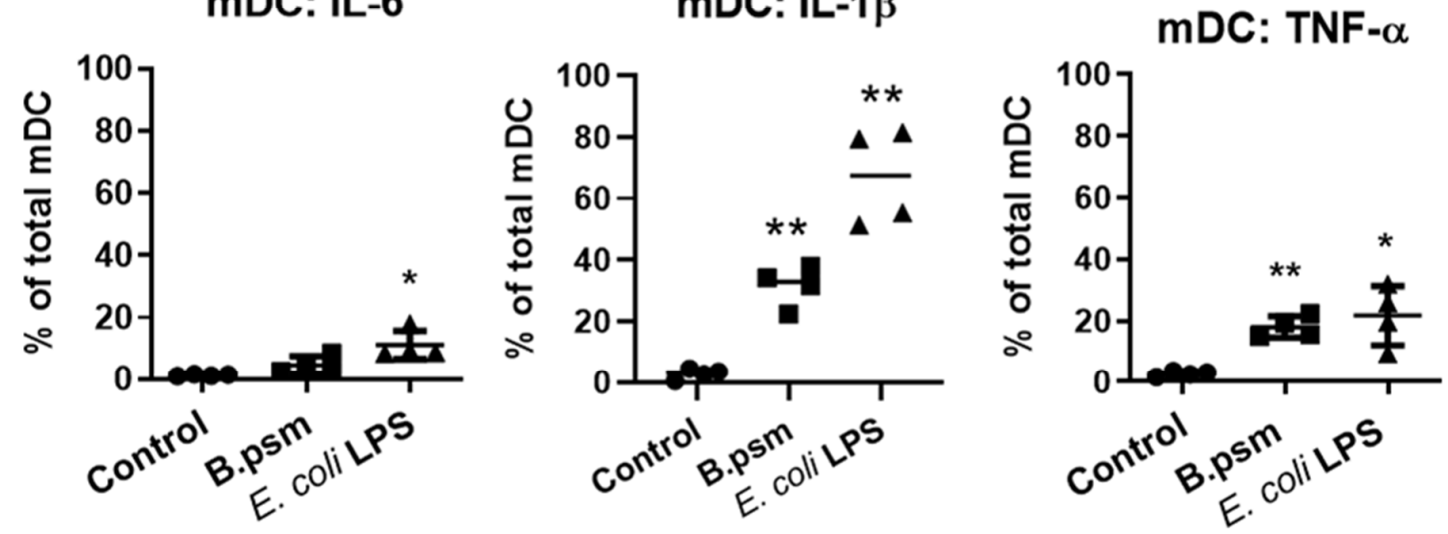

C
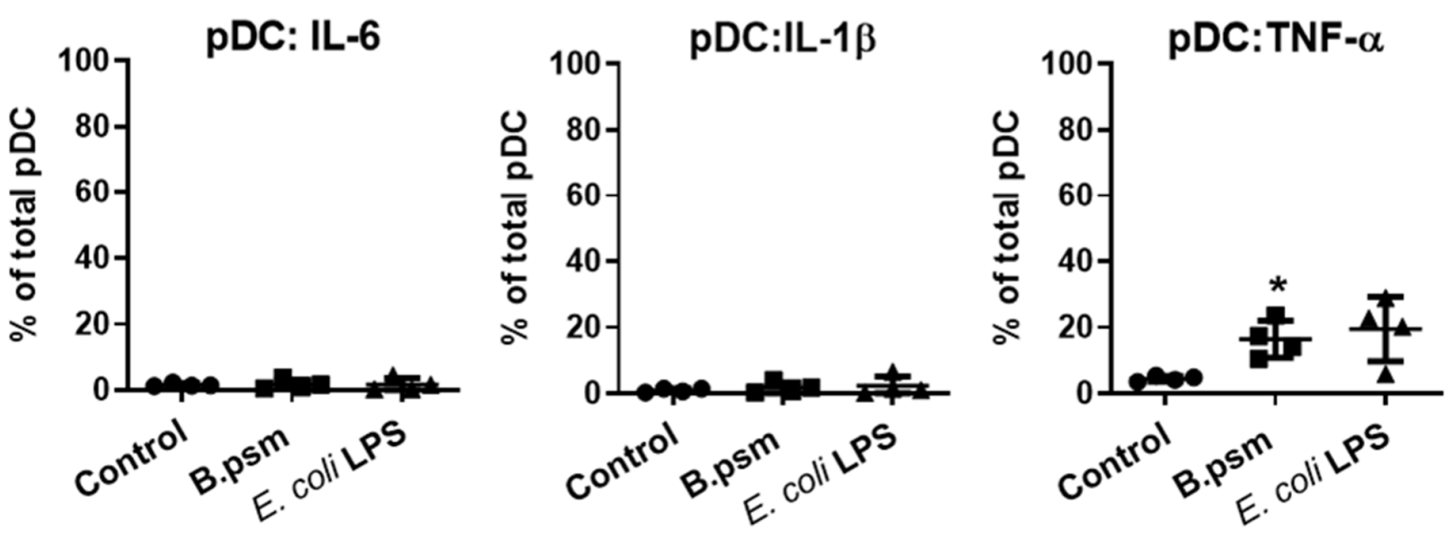

Figure 3. B. pseudomallei induces inflammatory cytokine production by myeloid and plasmacytoid DCs in healthy donors. (A) Amounts of secreted TNF- $\alpha$, IL-1 $\beta$ and IL-6 in supernatants of enriched DCs following 24-hour stimulation with B.psm or medium only are shown. Percentage of (B) mDCs and (C) pDCs expressing intracellular IL-6, IL-1 $\beta$ and TNF- $\alpha$ following 3-hour stimulation with B.psm, E. coli LPS or medium only. MFI of TNF- $\alpha$ in mDCs vs. pDCs after 3-hour stimulation with untreated control, $10^{6} \mathrm{CFU} / \mathrm{mL}$ B.psm or $100 \mathrm{ng} / \mathrm{mL}$ E. coli LPS was also determined. Bars represent mean values \pm SEM from three (A) or four $(\mathbf{B}, \mathbf{C})$ independent experiments. A one-way (ANOVA) followed by a Dunnett's multiple comparisons test was used to compare experimental groups to the medium only control. ${ }^{*} p<0.05,{ }^{* *} p<0.01,{ }^{* * * *} p<0.0001$. 


\subsection{DCs Conditioned with B.psm Stimulate $C D 4^{+}$T-Cell Proliferation}

B.psm-stimulated DCs became activated and produced cytokines; therefore, we next assessed their ability to induce T-cell proliferation. To enable this, we isolated and purified syngeneic T cells ex vivo from PBMCs using the flow process depicted in Figure 4, which yielded, using flow cytometry, a CD3+ fraction of $94 \%$ purity comprising $68 \% \mathrm{CD} 4+$ and $24 \%$ CD8+ T cells. Enriched DCs (combined $\mathrm{mDC}$ s and pDCs) were cultured with B.psm for $20 \mathrm{~h}$ and added at 5 or $10 \%$ to a syngeneic total T-cell population for 7 days. The $\mathrm{T}$ cells cultured with anti-CD3+ anti-CD28 were used as a positive control. The T cells subsequently acquired on the flow cytometer were divided from the total on the basis of a low expression of CTV. These CD4+CTV low or CD8+ CTV low were proliferated cells that were selected for further analysis (Figure 5A). The cell activation markers, CD45RA and CD45RO, were used to confirm the naïve or activated/memory status, respectively, within the divided and undivided cells (Figure 5A). In the left hand panel of Figure 5A, it can be seen that the divided CD4+ T cells activated non-specifically with anti-CD3+ antiCD28, or specifically activated with B.psm-pulsed DC, became predominantly CD45RO+ (87-92\% activated) compared with the unstimulated controls; the right hand panels of Figure 5A show that the divided CD8+ T cells could be non-specifically activated (73\%) with anti-CD3+ anti-CD28, but were not activated in response to B.psm-conditioned DCs. The undivided $\mathrm{CD} 4+\mathrm{T}$ cells had a much lower activation that did not vary greatly whether they were unstimulated, stimulated with anti-CD3+ anti-CD28 or specifically with B.psmpulsed DCs (60\% CD45RO+/19.6\% CD45RA; 45.7\% CD45RO+/33.1\% CD45RA; 59.5\% $\mathrm{CD} 45 \mathrm{RO}+/ 24.8 \% \mathrm{CD} 45 \mathrm{RA})$, respectively; therefore, these responses were not accepted as specific. The undivided CD8+ cells were less than $35 \%$ activated in any condition.

The B.psm-conditioned DCs added at $5 \%$ induced $\mathrm{CD}^{+}{ }^{+} \mathrm{T}$-cell proliferation, which was significantly higher than the untreated control after a 7-day culture (Figure 5B). Conversely, the B.psm-pulsed DCs added at either 5 or $10 \%$ did not significantly induce CD8 ${ }^{+}$T-cell proliferation (Figure $5 \mathrm{C}$ ). The majority of the $\mathrm{CD}^{+} \mathrm{T}$ cells that proliferated in response to B.psm treatment were CD45RO $\mathrm{R}^{+}$, indicating the activation of these cells (Figure 5A). Thus, by enriching for the DC population in human PBMCs, we have achieved an ex vivo priming of a CD4+ T-cell response to heat-killed B.psm.

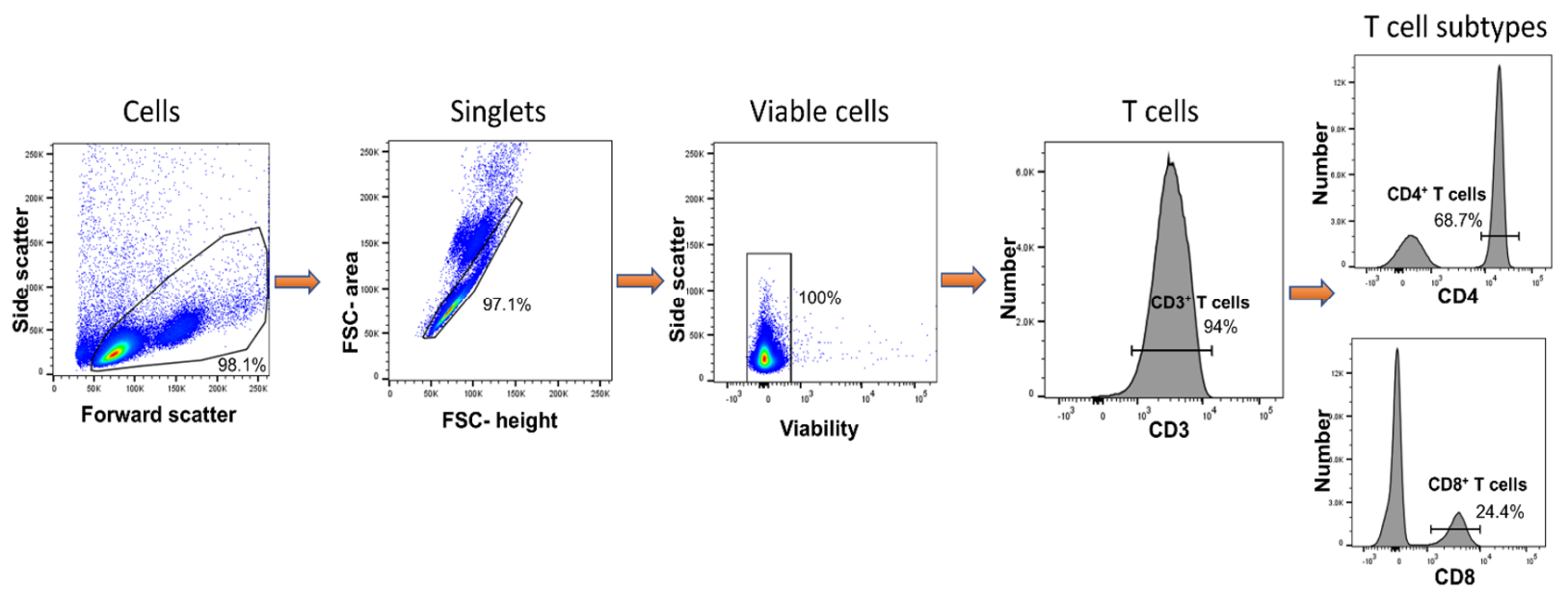

Figure 4. Purification of Total $\mathrm{CD} 4^{+}$and $\mathrm{CD} 8^{+} \mathrm{T}$ cells from fresh PBMCs. Total T cells were purified on day 0 from PBMCs and identified using side/forward scatter properties, doublet discrimination and total viable cells. Total CD3 ${ }^{+} \mathrm{T}_{\text {cells }}$ were subdivided using CD4 and CD8 markers to distinguish between $\mathrm{CD} 4^{+}$and $\mathrm{CD} 8{ }^{+} \mathrm{T}$ cells, respectively. 
A

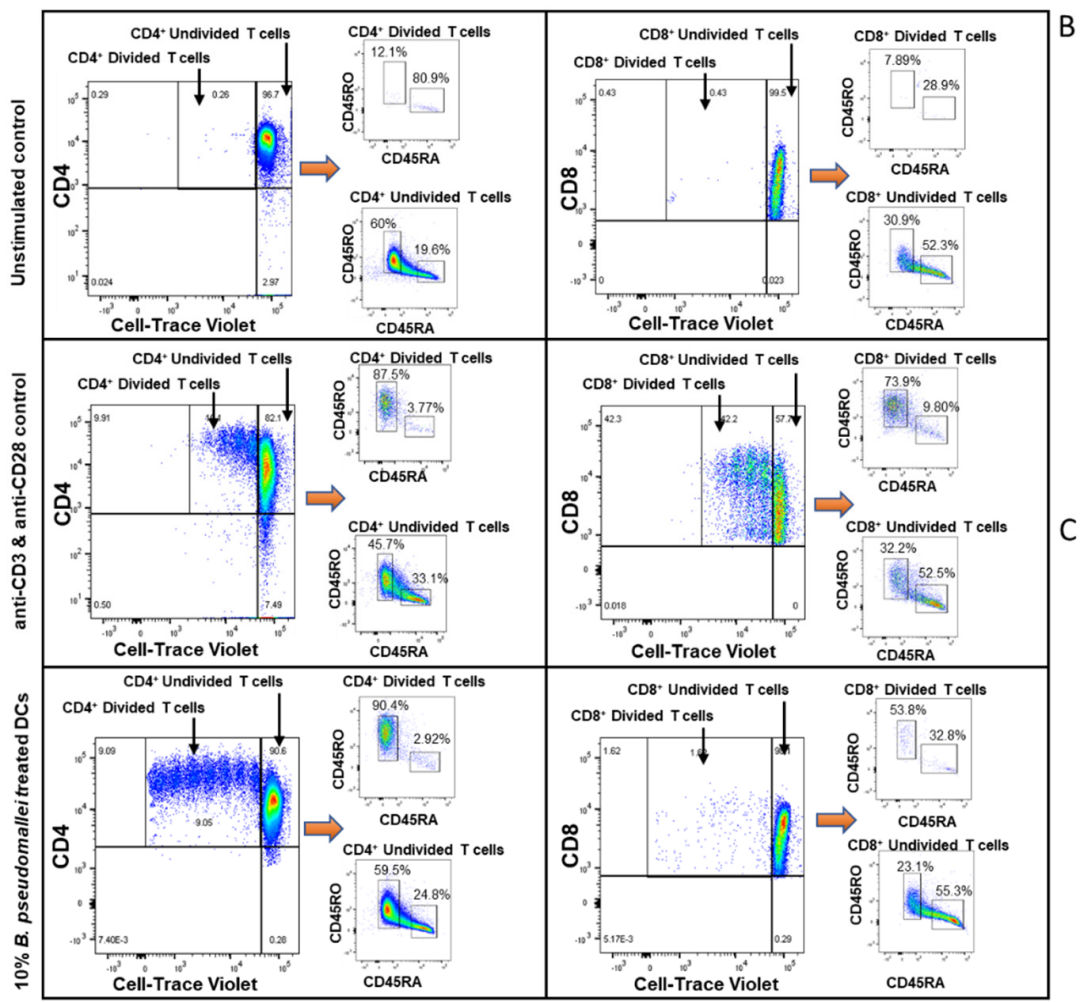

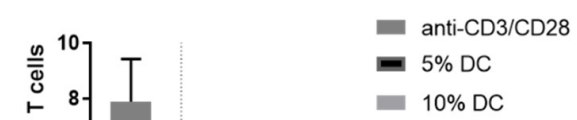

$10 \% \mathrm{DC}$
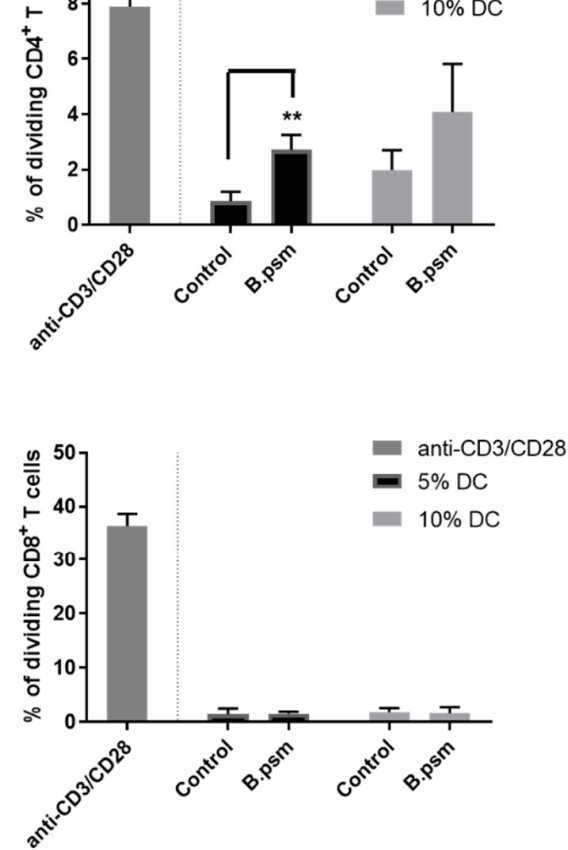

Figure 5. B. pseudomallei stimulates a primary $\mathrm{CD}^{+} \mathrm{T}$ helper cell response in healthy donors. Enriched DCs were stimulated for $20 \mathrm{~h}$ with B.psm or medium alone (control). $2.5 \times 10^{5}$ syngeneic total $\mathrm{T}$ cells were then co-cultured with either $5 \%$ or $10 \%$ stimulated DCs for 7 days. Anti-CD3 and anti-CD28 were used as a positive control for T-cell stimulation. (A) T cells subsequently acquired on the flow cytometer were divided from the total on the basis of low expression of CTV. These $\mathrm{CD} 4+\mathrm{CTV}^{\text {low }}$ or CD8+ CTV ${ }^{\text {low }}$ were proliferated cells and selected for further analysis. Cell activation markers, CD45RA and CD45RO, were used to confirm naïve or activated/memory status, respectively, within the divided and undivided cells. Examples are given of unstimulated (top panels), positive stimulation with anti-CD3 and anti-CD28 (middle panels) or $10 \%$ DC-treated with B.psm (bottom panels) $\mathrm{CD}^{+}$and $\mathrm{CD}^{+} \mathrm{T}$ cells after 7-days culture. Expression of cell-surface markers CD45RO and CD45RA were used to define activation status of proliferated vs. non-proliferated T cells. Pooled data showing the $\%$ of proliferated (B) $\mathrm{CD}^{+}$or $(\mathbf{C}) \mathrm{CD}^{+} \mathrm{T}$ cells after 7 days incubation with 5 or $10 \%$ of DCs stimulated with or without B.psm or with anti-CD3 and anti-CD28. Bars represent mean values \pm SEM from three independent experiments. A Student's paired $t$-test was used to compare the untreated control to B.psm group. ${ }^{* *} p<0.01$.

\section{Discussion}

We have demonstrated that heat-killed B.psm activates both human mDCs and pDCs enriched from fresh PBMCs of healthy individuals and induces their production of proinflammatory cytokines TNF- $\alpha$ and IL-1 $\beta$. While the mDCs were more strongly activated than the pDCs, with a greater expression of costimulatory molecules and IL- $1 \beta$, both subsets showed a significant up-regulation of MHC-II and produced TNF- $\alpha$ in response to B.psm. Furthermore, the combined $\mathrm{mDC}$ and $\mathrm{pDC}$ fractions stimulated with heat-killed B.psm induced a proliferation of syngeneic $\mathrm{CD}^{+} \mathrm{T}$ cells in vitro to form a small sub-population of memory $\mathrm{T}$ cells. This may reflect the in vivo situation where the current paradigm is that small populations of memory $\mathrm{T}$ cells, which are not long-lived in healthy humans, are maintained by repeated recruitment from the longer-lived naive pool [10], enabling the immune system to have the capacity to respond to a myriad of different antigens and insults.

Our study used modest volumes of blood from single healthy donors to examine the immune response to B.psm, which sets it apart from previous studies using bulk buffy coats. We chose to enrich the DCs from PBMCs using a negative selection protocol because, although positive selection gave higher purity, the negatively enriched DCs survived longer 
in culture. Conveniently, the use of negatively selected DCs allowed us to study both DC subsets simultaneously and build on previous work suggesting that pDCs as well as mDCs are directly involved in the immune response to B.psm [7]. Interestingly, both CD4 ${ }^{+}$and $\mathrm{CD}^{+} \mathrm{T}$-cell responses were induced when whole PBMCs from recovered melioidosis patients were cultured with B.psm or purified proteins, suggesting that protective memory involves both T-cell types [11]. It will be important in the future to further dissect the specific functions of $\mathrm{mDCs}$ and $\mathrm{pDCs}$ in promoting protective immune responses to B.psm to develop more effective vaccines and therapies.

DCs have been used in the clinic to treat cancer and autoimmune conditions [12]. More recently, pDCs were used to treat patients with metastatic melanoma by loading them with tumour antigens to induce type I interferon expression [13]. However, extensive clinical use of DCs is restricted by the low numbers of DCs present in peripheral blood and the ex vivo loading of DCs with an antigen for vaccine administration is not feasible in terms of cost, labour and the need for tailored therapy for each patient. The targeting of an antigen to DCs in vivo using antigen-antibody complexes to DC cell-surface receptors such as CD205 may prove feasible [14]. Live B.psm are taken up by DCs and induce the maturation and migration of immature DCs in vitro [15]. Although the interaction with the host cell differs for live and killed bacteria, the ability of DCs to become activated by heat-killed B.psm and our demonstration that these activated DCs can, in turn, activate even unprimed T cells, underlines the significant role for DCs in directing protective immunity to B.psm. By using cells from individuals with suspected melioidosis, it would be possible to apply the methods described herein to test for memory responses not only to killed bacteria, but also to bacterial sub-components, in order to identify those components required for protective immune responses and, thus, develop improved prophylactic strategies.

Furthermore, the methodology described here has the potential to be adopted more widely to screen vaccine candidates for other intracellular pathogens, where promoting a protective cell-mediated immune response is key.

\section{Conclusions}

In conclusion, we have shown that syngeneic $\mathrm{CD}^{+} \mathrm{T}$ cells can be primed ex vivo to respond with proliferation to B.psm-pulsed DC. The protocols used may be exploited to screen for sub-units of B.psm able to prime T cells and thus represent potential vaccine sub-units.

Author Contributions: Investigation D.R., L.D.; Methodology D.B., N.R.E.; Validation A.N.; Investigation P.H.: Resources G.C.C., J.L.P.; Conceptualisation E.D.W., S.C.K. All authors have read and agreed to the published version of the manuscript.

Funding: This research was funded by Dstl under contract DSTL/AGR/00254/01.

Institutional Review Board Statement: The study was conducted according to the guidelines of the Declaration of Helsinki. Ethical approval was obtained from the Health Research Authority UK and London Brent Research Ethics Committee (05.Q0405.71). Written informed consent was received from participants prior to inclusion.

Data Availability Statement: All data relating to this study is reported in the manuscript and figures.

Acknowledgments: We thank Rakesh Vora for his assistance with recruitment of healthy volunteers.

Conflicts of Interest: The authors declare no conflict of interest.

Author Summary: Melioidosis is an infectious disease endemic to south-east Asia and northern Australia caused by the bacterium Burkholderia pseudomallei. Melioidosis presents a significant public health threat because there is no effective vaccine or therapy, leading to a high mortality rate of between $10-50 \%$. We highlight the possibility of immune-based strategies targeting B. pseudomallei to better treat and prevent melioidosis. Specifically, we show that dendritic cells-the sentinel cells of the immune system-respond to B. pseudomallei in healthy individuals and, in turn, can orchestrate downstream protective immune responses. Thus, dendritic cells may be key players in the development of both vaccines and therapeutics for melioidosis as well as other bacterialdriven diseases. 
Content Includes Material Subject to @ Crown Copyright (2021), Dstl: This material is licensed under the terms of the Open Government Licence except where otherwise stated. To view this licence, visit http:/ / www.nationalarchives.gov.uk/doc/open-government-licence/version/3 (acessed on 19 August 2021) or write to the Information Policy Team, The National Archives, Kew, London TW9 4DU, or email: psi@nationalarchives.gov.uk.

\section{References}

1. Cheng, A.; Currie, B. Melioidosis: Epidemiology, pathophysiology, and management. Clin. Microbiol. Rev. 2005, 18, 383-416. [CrossRef] [PubMed]

2. Willcocks, S.J.; Denman, C.C.; Atkins, H.S.; Wren, B.W. Intracellular replication of the well-armed pathogen Burkholderia pseudomallei. Curr. Opin. Microbiol. 2016, 29, 94-103. [CrossRef] [PubMed]

3. Merad, M.; Sathe, P.; Helft, J.; Miller, J.; Mortha, A. The Dendritic Cell Lineage: Ontogeny and Function of Dendritic Cells and Their Subsets in the Steady State and the Inflamed Setting. Annu. Rev. Immunol. 2013, 31, 563-604. [CrossRef] [PubMed]

4. Parcina, M.; Wendt, C.; Goetz, F.; Zawatzky, R.; Zähringer, U.; Heeg, K.; Bekeredjian-Ding, I. Staphylococcus aureus-Induced Plasmacytoid Dendritic Cell Activation Is Based on an IgG-Mediated Memory Response. J. Immunol. 2008, 181, 3823-3833. [CrossRef] [PubMed]

5. Veckman, V.; Julkunen, I. Streptococcus pyogenes activates human plasmacytoid and myeloid dendritic cells. J. Leukoc. Biol. 2008, 83, 296-304. [CrossRef] [PubMed]

6. $\quad$ Piccioli, D.; Sammicheli, C.; Tavarini, S.; Nuti, S.; Frigimelica, E.; Manetti, A.G.; Nuccitelli, A.; Aprea, S.; Valentini, S.; Borgogni, E.; et al. Human plasmacytoid dendritic cells are unresponsive to bacterial stimulation and require a novel type of cooperation with myeloid dendritic cells for maturation. Blood 2009, 113, 4232-4239. [CrossRef] [PubMed]

7. Williams, N.L.; Morris, J.L.; Rush, C.M.; Ketheesan, N. Plasmacytoid dendritic cell bactericidal activity against Burkholderia pseudomallei. Microbes Infect. 2015, 17, 311-316. [CrossRef] [PubMed]

8. Elvin, S.J.; Healey, G.D.; Westwood, A.; Knight, S.C.; Eyles, J.E.; Williamson, E.D. Protection against Heterologous Burkholderia pseudomallei Strains by Dendritic Cell Immunization. Infect. Immun. 2006, 74, 1706-1711. [CrossRef] [PubMed]

9. Sarkar-Tyson, M.; Smither, S.J.; Harding, S.V.; Atkins, T.P.; Titball, R.W. Protective efficacy of heat-inactivated B. thailandensis, $B$. mallei or B. pseudomallei against experimental melioidosis and glanders. Vaccine 2009, 27, 4447-4451. [CrossRef] [PubMed]

10. Westera, L.; Drylewicz, J.; den Braber, I.; Mugwagwa, T.; van der Maas, I.; Kwast, L.; Volman, T.; van de Weg-Schrijver, E.H.; Bartha, I.; Spierenburg, G.; et al. Closing the gap between T-cell life span estimates from stable isotope-labeling studies in mice and humans. Blood 2013, 122, 2205-2212. [CrossRef]

11. Tippayawat, P.; Saenwongsa, W.; Mahawantung, J.; Suwannasaen, D.; Chetchotisakd, P.; Limmathurotsakul, D.; Peacock, S.J.; Felgner, P.L.; Atkins, H.S.; Titball, R.W.; et al. Phenotypic and Functional Characterization of Human Memory T Cell Responses to Burkholderia pseudomallei. PLoS Negl. Trop. Dis. 2009, 3, e407. [CrossRef]

12. Palucka, K.; Banchereau, J. Human dendritic cell subsets in vaccination. Curr. Opin. Immunol. 2013, 25, 396-402. [CrossRef] [PubMed]

13. Tel, J.; Aarntzen, E.H.; Baba, T.; Schreibelt, G.; Schulte, B.M.; Benitez-Ribas, D.; Boerman, O.C.; Croockewit, S.; Oyen, W.J.; van Rossum, M.; et al. Natural Human Plasmacytoid Dendritic Cells Induce Antigen-Specific T-Cell Responses in Melanoma Patients. Cancer Res. 2013, 73, 1063-1075. [CrossRef]

14. Tacken, P.J.; de Vries, I.J.M.; Torensma, R.; Figdor, C.G. Dendritic-cell immunotherapy: From ex vivo loading to in vivo targeting. Nat. Rev. Immunol. 2007, 7, 790-802. [CrossRef] [PubMed]

15. Williams, N.L.; Morris, J.L.; Rush, C.M.; Ketheesan, N. Migration of Dendritic Cells Facilitates Systemic Dissemination of Burkholderia pseudomallei. Infect. Immun. 2014, 82, 4233-4240. [CrossRef] [PubMed] 